\title{
Usages des technologies par les élèves professeurs : analyse à partir de questionnaires
}

\section{Philippe Le Borgne}

Laboratoire de didactique des mathématiques de Paris 7, FRANCE

philippe.leborgne@fcomte.iufm.fr

Jean-Paul Fallot

IUFM de Franche-Comté, FRANCE

jean-paul.fallot@fcomte.iufm.fr
Jean-François Lecas

Pôle AAFE, Esplanade Erasme, Campus universitaire, Dijon, FRANCE jean-francois.lecas@laposte.net

Agnès Lenfant

IUFM de Champagne-Ardenne, FRANCE

agnes.lenfant@reims.iufm.fr

Compte rendu d'expérience

\section{Résumé}

Cet article étudie l'appropriation et les usages des technologies chez les élèves professeurs français. La méthodologie utilisée est basée sur l'analyse de réponses à des questionnaires soumis aux professeurs stagiaires de quatre instituts universitaires de formation des maîtres. Les résultats obtenus tendent à prouver que les enseignants en formation sont aujourd'hui bien équipés et disposent de compétences réelles dans le cadre des usages personnels et professionnels en dehors de la classe. Cependant, il apparaît que ces compétences sont peu mobilisées dans le cadre des usages en classe.

\section{Abstract}

This article studies the appropriation and the uses of technologies by French student teachers. The methodology used is based on the analysis of answers to questionnaires submitted to the trainees of four "instituts universitaires de formation des maîtres" (teacher training schools). The results obtained tend to prove that outside the classroom trainee teachers are today well equipped and really competent as far as personal and professional uses of technologies are concerned. However, it appears that these skills are not taken advantage of in the presence of the pupils. 


\section{Usages des technologies par les élèves professeurs : analyse à partir de questionnaires}

L'usage des technologies est largement encouragé dans le système éducatif français ${ }^{1}$ et de nombreux travaux de recherche ont mis en évidence les contributions possibles des technologies de l'information et de la communication ( $\mathrm{TIC}^{2}$ ) aux apprentissages des élèves (Laborde et Capponi, 1994; Linard, 1996) et au renouvellement des pratiques scolaires (Baron, Caron et Harrari, 2005; Bellemain et Capponi, 1992). Cependant, certaines études ${ }^{3}$ montrent les réticences de nombreux enseignants à s'approprier les outils informatisés, à les intégrer dans leur démarche professionnelle. L'étude des usages des technologies par les enseignants paraît aujourd'hui essentielle (Baron, Bruillard et Levy, 2000; Monaghan, 2004; Rinaudo, 2001). Nous proposons de l'aborder à travers l'étude de la population des professeurs stagiaires des instituts universitaires de formation des maîtres (IUFM). Ces futurs professeurs d'école (PE) et de lycées et collèges (PLC) ont réussi le concours d'aptitude au professorat et bénéficient du statut de professeur stagiaire. Leur formation professionnelle initiale est fondée sur une articulation entre des formations à l'IUFM et des stages en responsabilité en établissement scolaire au cours desquels ils sont accompagnés par des maîtres de stage et des formateurs des IUFM.

Le système éducatif français connaît actuellement un renouvellement important des enseignants et son évolution va être conditionnée par la capacité des nouveaux professeurs à élaborer des techniques professionnelles adaptées à la diversité des situations d'enseignement. Il paraît donc essentiel d'étudier leurs rapports aux $\mathrm{TICE}^{4}$. Selon des recherches récentes (Baillat et Vincent, 2003), ce public est mieux outillé et connecté que l'ensemble de la population, et il est conscient de la nécessité d'une formation en TICE. Par ailleurs, plusieurs résultats montrent que l'année de stage est déterminante dans la formation des pratiques qui deviennent rapidement stables et cohérentes (Lenfant, 2003).

Une équipe constituée de chercheurs de quatre IUFM bénéficiant du soutien de l'Institut national de la recherche pédagogique s'est proposé de considérer le problème de l'intégration des TICE dans l'enseignement en étudiant la population des professeurs stagiaires (Lagrange, Lecas et Parzysz, sous presse) afin de mieux connaître les rapports des nouveaux enseignants avec les technologies et de comprendre l'évolution des rapports avec celles-ci pendant la formation et les premiers temps d'exercice du métier.

\section{Problématique}

Nous cherchons à identifier les usages des technologies que les professeurs stagiaires intègrent facilement, les points de résistance et les déterminants qui les sous-tendent. Nous distinguons trois cadres d'usage des technologies correspondant à différents contextes d'activité : i) les activités professionnelles non directement liées à la classe: recherche d'informations, échanges entre pairs; ii) le travail du professeur " en différé " relatif aux apprentissages de ses élèves: conception de séances d'enseignement et production de documents, évaluation des apprentissages des élèves, etc.; iii) l'utilisation en classe. Nous considérons les usages dont l'objectif est de soutenir des apprentissages disciplinaires ou méthodologiques, tirant parti de logiciels disciplinaires ou généraux.

Dans ces trois cadres, les technologies viennent «instrumenter » l'activité du professeur. Les usages dans le premier cadre et certains usages dans le deuxième utilisent des outils et démarches similaires à ceux rencontrés dans d'autres professions (ex.: production de documents) tandis que d'autres usages dans les deuxième et troisième cadres mettent en jeu des outils et démarches spécifiques à l'enseignement (ex.: étude d'un problème avec un logiciel de géométrie dynamique). Dans le troisième cadre, cette instrumentation s'articule avec une activité instrumentée des élèves.

Notre réflexion est problématisée autour de deux hypothèses:

Premièrement, il existerait au sein de chaque cadre un contraste entre des usages se développant "naturellement » et d'autres posant plus de difficultés. Certains déterminants de cette hétérogénéité peuvent être recherchés dans les domaines suivants:

- Représentations de l'enseignement et des technologies. Un professeur stagiaire ne développerait des usages des TICE que s'ils lui semblent conformes à l'idée qu'il se fait de l'enseignement de sa discipline et du rôle que peuvent y jouer les TICE;

- Contraintes de l'exercice du métier et normes associées. Par exemple la disponibilité du matériel et l'accès aux salles, le temps de préparation, la complexité de l'organisation pédagogique et de sa mise en œuvre, le respect des programmes;

- Formation reçue au cours de l'année de stage et lors des études antérieures. Elle agirait sur les représentations, la perception des contraintes du métier et l'intégration des normes associées.

Deuxièmement, pour un outil donné, il existerait des écarts entre les usages dans les différents cadres d'activités. Ils seraient révélateurs de ruptures qualitatives: plus grande complexité de mise en œuvre, représentations différentes, résistances dues aux normes et 
contraintes spécifiques à chaque cadre. Ces écarts seraient associés à des tâches professionnelles de nature différente. L'enseignant utilisant les technologies dans les deux premiers cadres réalise une tâche individuelle peu contrainte. Dans le cadre de la réalisation en classe, la tâche est essentiellement coopérative (Chevallard, 1997). Sa viabilité nécessiterait une dévolution aux élèves de la pratique des outils et un changement de posture du professeur.

L'investissement réalisé par l'Éducation nationale dans le domaine des TICE ne se justifierait pas si les enseignants utilisaient les technologies seulement en dehors de la classe. Il s'agit donc de caractériser les écarts qualitatifs qui constituent un obstacle au développement des usages en classe et de rechercher les facteurs qui favorisent la transition d'un cadre à l'autre.

\section{Méthodologie}

Notre travail s'est appuyé sur quatre questionnaires. Trois d'entre eux sont destinés à piloter les dispositifs de formation aux technologies. Il nous a semblé pertinent de travailler sur les données issues des enquêtes, car elles couvrent assez bien l'ensemble des informations nécessaires avec un taux de réponses utiles satisfaisant et permettent des recoupements. Cependant, certaines informations relevant de l'usage en classe n'apparaissaient pas dans ces questionnaires. Plutôt que d'alourdir des outils déjà complexes et de passation longue, nous avons préféré compléter ces informations par un quatrième (Besançon), conçu spécifiquement pour cette recherche.

Pour l'IUFM de Reims, l'étude est basée sur des questionnaires en ligne proposés de 1999 à 2003 en fin de formation aux PE et aux PLC pour pointer les connaissances en TIC, identifier les usages et les lieux d'utilisation et repérer les représentations des stagiaires dans l'utilisation des TICE. En 2003, 260 PE sur 335 (78\%) et 108 PLC sur 371 (29\%) ont répondu.

L'IUFM de Dijon utilise un questionnaire depuis 1999, obligatoirement renseigné en ligne par l'ensemble des PE et des PLC début septembre. Il sert à orienter les dispositifs de remédiation pour mettre à niveau les stagiaires qui ne possèdent pas les compétences du C2i niveau $1^{5}$. Il porte essentiellement sur l'équipement des stagiaires et sur leurs compétences déclarées en TIC. Les données présentées ici portent sur deux études : la première a regroupé en 2003 les réponses des 374 PLC, la seconde exploite les résultats des 518 questionnaires PE dépouillés en 2004.

L'IUFM d'Orléans-Tours a soumis un questionnaire en octobre 2003 à un échantillon de $100 \mathrm{PE}$ puis à nouveau au mois de juin suivant; 43 réponses ont été recueillies. Les résultats nous informent sur l'équipement personnel des stagiaires, leurs compétences en informatique et les types d'activités TICE pratiquées en classe. Cette méthodologie a contribué à percevoir des facteurs expliquant l'évolution des usages sur l'année et à repérer les éléments déclencheurs pouvant favoriser une pratique des TICE.

Pour l'IUFM de Besançon, le questionnaire a été proposé au printemps 2003: 178 PE sur 323 (55\%) et 120 PLC sur 348 (34\%) y ont répondu en ligne. Il renseigne sur l'équipement des stagiaires et des lieux de stage, et les utilisations personnelles et professionnelles des TICE. Il informe également sur l'acquisition des compétences utiles aux usages des technologies et sur le rôle de la formation.

L'hétérogénéité des résultats obtenus ne permet pas de comparer directement les IUFM. Cependant, des rapprochements effectués entre ces données ont mis en évidence les concordances ou différences dans les résultats obtenus d'un IUFM à l'autre. Certains résultats isolés, intéressant notre problématique, ont été retenus. Par ailleurs, les questionnaires mis en place plusieurs années de suite (Reims et Dijon), permettent de relever des évolutions; elles seront mentionnées lorsque les comparaisons sont pertinentes.

\section{Résultats}

\section{L'équipement personnel des professeurs stagiaires}

\section{Ordinateur}

Le taux d'équipement en ordinateur dépasse $75 \%$ en début d'année de stage dès $2003^{6}$ dans toutes les enquêtes. Certaines données (Reims, Besançon) montrent une progression de l'équipement en cours d'année.

\section{Accès à Internet}

Pour Reims, le taux d'accès à domicile passe de $24 \%$ en 1999 à $76 \%$ en 2003 avec une augmentation entre début et fin de l'année. Cette enquête pointe une spécificité des PLC littéraires avec un taux d'accès ( $81 \%$ ) supérieur aux PE (77\%) et aux PLC scientifiques $(66 \%)$. Besançon indique un taux d'accès à Internet de $72 \%$ pour les PLC et de $69 \%$ pour les PE.

\section{Les usages généraux de l'informatique}

\section{Manipulation de l'ordinateur}

Les compétences de base (ex.: mettre en route un ordinateur, rechercher de façon simple une information) sont acquises en début d'année par les PLC de Dijon. L'organisation d'un espace de 
travail avec copie et classement des dossiers est également acquise avec une disparité homme-femme (90\% vs $77 \%$ ). Les procédures d'enregistrement des données (84\% à Reims) témoignent de compétences élevées, les compétences de base et la possession d'un ordinateur étant largement corrélées (Orléans-Tours).

\section{Réseaux et navigation}

Comparées aux objectifs du C2in1, les compétences concernant les réseaux sont plutôt faibles. Seulement $25 \%$ des PE de Dijon savent partager une ressource et $41 \%$ savent qu'un ordinateur peut être raccordé à un réseau; les réponses concernant l'utilisation de moteurs de recherche semblent paradoxales : $95 \%$ des stagiaires déclarent en utiliser pour trouver un site pertinent, mais seuls $40 \%$ utilisent les opérateurs booléens. Cependant, d'autres facteurs jouent certainement un rôle dans la pertinence de la recherche (détermination précise des mots clés, rang dans la requête, etc.).

\section{Messagerie et sites collaboratifs}

À Dijon, $83 \%$ des PE ont une adresse électronique ( $82 \%$ à Reims). L'utilisation de la messagerie semble se faire a minima pour certaines fonctions classiques: ajouter automatiquement une signature (44\%); ranger des messages dans des dossiers (63\%); envoyer un message avec un fichier joint (72\%). L'enquête d'Orléans-Tours précise que $20 \%$ des stagiaires n'utilisent pas la messagerie électronique. Tous les résultats sont cohérents : un stagiaire sur cinq n'est pas un utilisateur régulier, même si une adresse électronique lui est attribuée par l'institution. On ne relève pas de compétences affirmées pour des outils de communication asynchrone tels que forums ou listes de diffusion. Seuls $21 \%$ des stagiaires font la différence entre les forums de discussion modérés et non modérés, et $25 \%$ savent utiliser une liste de diffusion.

\section{Aspects éthiques et juridiques}

Les données de Dijon indiquent une faible connaissance de la législation relative aux TICE, les taux de réponse variant de $40 \%$ pour les aspects juridiques et éthiques à $26 \%$ pour la connaissance des règles définies dans les chartes d'usage. Ceci peut être dû à des connaissances insuffisantes ou à une faible maîtrise du lexique spécialisé. Ce fait nous amène à nous interroger sur l'impact des formations préalables et les risques encourus par les stagiaires dans l'utilisation de certains logiciels.

\section{Logiciels généraux, production de documents}

Les compétences élémentaires relatives à l'utilisation du traitement de texte sont élevées ( 86 \%) en début d'année scolaire chez les PE (Dijon et Besançon), confirmant les scores observés chez les PLC. Concernant le tableur, les connaissances des stagiaires semblent assez faibles (Dijon, $57 \%$ ). Ces résultats sont confirmés par Reims et Besançon.

Dijon relève que les $\mathrm{PE}$ disposent de peu de compétences dans la production de documents multimédias et le travail sur l'image (création d'un site: $7 \%$, d'un diaporama: $25 \%$ ), résultats confirmés par Besançon. Les résultats sont meilleurs à Reims (66\% en 2001), mais en baisse constante. Ce fait serait à relier à la mise en place d'un nouveau dispositif de formation n'incluant plus la formation à la création d'un site Web.

De nouvelles compétences pour créer et transformer les images, liées à la large diffusion de numériseurs et d'appareils photo numériques, sont requises pour préparer des documents pour la classe. Les résultats de Dijon montrent qu'elles sont insuffisantes : numériser une image à l'aide d'un numériseur (50\%); choisir le format d'enregistrement de l'image numérisée $(12,5 \%)$.

\section{La réalité et la genèse des compétences}

Concernant les usages personnels et professionnels, deux tendances émergent:

i) le traitement de texte est utilisé pour la création de documents courts alors que la gestion des documents longs semble peu maîtrisée; ii) des faiblesses différenciées apparaissent selon les disciplines dans le traitement des images numériques et le tableur.

Près des deux tiers des PE de Dijon affirment ne pas avoir suivi de formation informatique alors que l'Université de Dijon intègre un module informatique dans les premiers cycles et licences depuis plus de 15 ans. Nous retrouvons ce sentiment d'absence de formation dans trois enquêtes de Reims. Le tableau suivant indique où les stagiaires de Besançon déclarent avoir commencé l'informatique.

\begin{tabular}{|c|c|c|c|c|c|}
\hline & $\begin{array}{c}\text { Hors dispositif } \\
\text { de formation }\end{array}$ & Àl'université & Au collège & Au lycée & Àl'IUFM \\
\hline PLC & 49 & 17 & 10 & 6 & 4 \\
\hline PE & 39 & 23 & 16 & $/$ \\
\hline
\end{tabular}

Tableau 1. Début en informatique déclaré par les stagiaires de Besançon (en \%) 
Selon ces enquêtes, l'autodidaxie paraît donc être la principale modalité d'apprentissage en TIC.

Concernant les PLC, la découverte de l'existence de logiciels pédagogiques repose plutôt sur les formateurs IUFM, alors que pour les $\mathrm{PE}$, les collègues stagiaires jouent un rôle prépondérant (voir le Tableau 2).

\begin{tabular}{|l|c|c|}
\hline & PE & PLC \\
\hline Formateurs IUFM & 21 & 42 \\
\hline Maîtres formateurs et maitres de stage & 4 & 20 \\
\hline Collègues et amis & 37 & 35 \\
\hline Presse & 20 & 14 \\
\hline
\end{tabular}

Tableau 2. Découverte par les PE et les PLC (Besançon) de l'existence de logiciels pédagogiques (en \%)

Il existe en fait un large éventail de sources d'information et de formation. L'IUFM aurait logiquement dû apparaitre en premier, compte tenu de ses missions. Ceci se vérifie uniquement chez les PLC. Dans ce cas, les formateurs IUFM apparaissent sans doute les mieux placés pour présenter les logiciels lors de modules spécifiques à la discipline. D'une manière générale, les collègues constituent une ressource importante.

\section{La préparation de la classe}

\section{Connaissance des potentialités des TICE}

L'enquête de Reims renseignait sur les niveaux de connaissance des logiciels « reconnus d'intérêt pédagogique »?. Ceci est un indicateur de l'insertion des TICE dans la discipline. En 2000, $46 \%$ de l'échantillon ne connaissait pas ces logiciels. On notait des disparités importantes entre PE (39\%), PLC littéraires (39\%) et PLC scientifiques (64\%). En 2003, le nombre global des stagiaires ne connaissant pas ces logiciels (37\%) diminuait légèrement, mais les PLC littéraires déclarant ne pas les connaitre représentaient plus de $66 \%$, contre $26 \%$ pour les PLC scientifiques. De tels renversements ne peuvent résulter que des informations transmises dans les groupes de formation. Parmi ceux qui les connaissaient, un sur deux mettait l'accent sur la lourdeur des préparations nécessaires à leur usage en classe. Une question concernait le degré d'acceptation d'un travail réalisé sur traitement de texte par les élèves; en 2003, la grande majorité ( $86 \%$ ) des stagiaires interrogés acceptait ce support, mais $40 \%$ décourageaient les élèves de recommencer.

Recherche d'informations pédagogiques sur Internet

À Reims (2000), la moitié des stagiaires déclarait avoir recours à Internet pour rechercher des informations et un tiers y trouver des préparations « toutes faites ». Ces taux progressent régulièrement : en 2003,78\% des stagiaires affirmaient y rechercher des informations et $57 \%$, des préparations. Ces augmentations sont à mettre en relation avec le taux de raccordement personnel et le développement du nombre de sites Web à destination des enseignants. Cette tendance appelle deux remarques : i) les documents trouvés sur le Web présentent un intérêt pédagogique et une qualité très variables. Compte tenu de la faiblesse des compétences constatées chez les stagiaires dans le domaine de la recherche documentaire et dans celui de la connaissance de la législation informatique, on peut s'inquiéter quant à la pertinence de l'utilisation de ces documents en classe; ii) cette utilisation d'Internet est davantage orientée vers la réception que vers la collaboration. Orléans-Tours pointe, en effet, que les échanges entre stagiaires demeurent peu fréquents (40\%) malgré le contexte favorable (année de formation) et les services offerts par l'Institut (messagerie, liste de diffusion, etc.).

Production de documents et utilisation de logiciels disciplinaires À Orléans-Tours, $90 \%$ des stagiaires déclaraient élaborer leurs fiches de préparation et documents professionnels sur ordinateur, mais seulement $60 \%$ s'en servaient pour produire des documents à destination des élèves. Ces données sont cohérentes avec les faibles compétences à produire des documents complexes et à intégrer des images.

L'utilisation de logiciels disciplinaires pour la préparation de la classe par les PLC est très minoritaire (17 \% à Reims en 2003) et reste stable pour les PE entre 2000 et 2003 (environ $10 \%$ ). Les PLC scientifiques utilisent davantage de logiciels spécifiques que leurs collègues littéraires (57 \% vs $8 \%$ en 2003).

\section{Les usages en classe}

L'accès à des moyens informatiques sur les lieux de stage Besançon montre que l'accès au matériel informatique pour l'enseignement est généralement possible dans les établissements scolaires (secondaire: $82 \%$, primaire : $66 \%$ ). Un tiers des stagiaires d'Orléans-Tours rapporte des difficultés liées au manque d'ordinateurs.

\section{L'utilisation en classe}

À Besançon, $56 \%$ des PE et $57 \%$ des PLC affirment avoir utilisé les TICE en classe; Reims mentionne des taux légèrement supérieurs avec une progression au cours des trois dernières années (PE de 60 à $64 \%$; PLC de 63 à $79 \%$ ). À OrléansTours, $75 \%$ des PE déclarent avoir eu recours à l'ordinateur en classe et $20 \%$ sont en passe de systématiser l'outil. Le 
traitement de texte est le plus couramment utilisé; le navigateur est peu employé ( $25 \%$ en cycle $\left.3^{8}\right)$; le courrier électronique, l'insertion d'images et la recherche documentaire sur $\mathrm{CD}$-ROM ne sont pas intégrés aux pratiques. Concernant le traitement de texte, la majorité des usages a lieu en cycle 3. L'objectif est d'enseigner des fonctionnalités de base, avec la mise en forme d'écrits personnels ou la réalisation d'un journal d'école. Sur des échantillons plus larges, Reims confirme l'usage du traitement de texte en classe par les PE (51\%). Les PLC scientifiques privilégient l'utilisation de logiciels disciplinaires $(66 \%)$ et les littéraires orientent leurs élèves vers la recherche de documents sur Internet (39\%). L'usage du tableur n'évolue pas ( $3 \%$ en 2000 et $5 \%$ en 2003) et concerne uniquement des PLC scientifiques.

\section{Les déterminants des usages}

\section{Sources d'information}

Concernant la présentation de logiciels pédagogiques, l'IUFM devrait logiquement être le premier nommé compte tenu de ses missions. Besançon indique que ceci se vérifie seulement chez les PLC (43\%), les PE étant informés principalement par leurs collègues et par leurs amis (37 \%).

On peut penser qu'en stage la source d'information est plutôt une personne ressource. Tout se passe comme si les TICE restaient, dans les établissements, l'affaire d'un nombre limité d'enseignants. Ces personnes ressources sont moins accessibles dans le premier degré que dans le second et les PE doivent se tourner vers des sources extérieures. Ces données sont cohérentes avec les résultats d'Orléans-Tours (le premier facteur est l'environnement professionnel, les programmes et l'intérêt personnel venant en second) et de Besançon où les stagiaires prennent conseil auprès de collègues (45\%), d'amis $(36 \%)$ et de formateurs IUFM (30\%).

\section{Motivations et représentations}

Les questionnaires montrent que les stagiaires ne considèrent pas que les usages en classe des TICE sont réservés aux experts. Ils se déclarent prêts, si les conditions matérielles le permettent, à les mettre en œuvre dès le début de leur carrière (PE $97 \%$; PLC scientifiques $94 \%$; PLC littéraires $81 \%$ ).

Parmi les avantages associés à l'usage des TICE, ils indiquent la dimension culturelle ( $80 \%$ ), les apports des TICE aux apprentissages disciplinaires $(50 \%)$ et la vision de l'ordinateur comme aide à la mobilisation de l'attention des élèves (40\%).
Parmi les usages possibles, deux stagiaires sur trois privilégient la remédiation et un sur deux, l'apprentissage ou l'entraînement. Seul un sur trois imagine une utilisation avec la classe entière. Ils privilégient donc des organisations de travail où l'ordinateur sert à mettre les élèves en activité sans mobiliser le professeur.

\section{Discussion}

Notre recherche visait à mettre en évidence l'usage des TICE chez les professeurs stagiaires en formation professionnelle et à repérer les obstacles susceptibles de s'opposer à des conditions favorables au développement d'usages professionnels.

Nous avons choisi de nous interroger sur la formation initiale en rapport avec la contribution possible des TICE aux apprentissages des élèves. Cependant, notre méthodologie ne permet pas de décrire précisément les usages et nous autorise seulement à établir des hypothèses. L'impact des représentations, les contraintes du terrain, la fréquence des usages, les rapports de ceux-ci avec la formation, l'instrumentation de la classe, etc. devront être étudiés à l'aide d'une autre méthodologie basée par exemple sur des entretiens et des observations dans les classes. L'analyse des réponses obtenues à des questionnaires déclaratifs limite donc notre étude au relevé des écarts et à la mention de grandes tendances.

Ces études confirment le fait que la population des nouveaux enseignants est généralement bien équipée en matériel informatique et connectée au réseau Internet (Baillat et Vincent, 2003). Dans le cadre des usages non directement liés à la classe, ce résultat rejoint ce qui est constaté chez les jeunes professionnels, quel que soit le secteur d'activité concerné. Cependant, nous devons nous garder de généraliser les résultats apportés par cette recherche puisque de nombreuses disparités ont pu être observées: homogénéité chez les PE aux cursus universitaires variés, vraisemblablement liée à leur parcours commun lors de la préparation aux concours à l'IUFM; hétérogénéité chez les PLC, sans doute liée aux parcours disciplinaires.

Dans le cadre des activités professionnelles non directement liées à la classe, les usages privilégiés sont essentiellement basiques: production de documents simples, recherche d'informations via Internet sans utilisation de critère de recherche performant. La fraction des professeurs stagiaires restant rétive à l'utilisation du courriel $(20 \%)$ est préoccupante. Beaucoup d'informations dans le cadre des formations sont transmises par le biais de listes de diffusion et risquent d'échapper à une partie de la population des stagiaires; c'est aussi un levier sur lequel les IUFM peuvent 
agir pour enrichir les compétences des usagers. Au moment où les questionnaires ont été passés, le C2inl n'en était quà sa mise en place à l'université, il conviendra donc d'être attentif aux modifications que provoquera sa généralisation ainsi quà celles dues à l'introduction du C2in2 (C2i niveau 2 - Enseignant, n.d.) dans les IUFM. Les aspects éthiques et juridiques sont assez peu connus des stagiaires. S'ils savent qu'il existe une législation dans ce domaine, ces connaissances ont peu d'impact sur les pratiques. De ce fait, l'utilisation de certains logiciels peut comporter des risques sérieux pour une utilisation personnelle qui peuvent devenir graves en cas d'utilisation en classe.

Les professeurs stagiaires semblent volontaires pour envisager l'intégration des technologies dans leurs pratiques. Parallèlement, cette représentation est associée essentiellement à une dimension «culturelle» des TICE, incluant peu les apports possibles aux apprentissages disciplinaires et les usages en classe entière. Les stagiaires conservent-ils une représentation de la classe qui réfère à leur propre scolarité, scolarité durant laquelle ils semblent avoir eu peu de contact avec les technologies? Toujours est-il que l'investissement des TICE semble s'effectuer uniquement pour les préparations de documents personnels ou destinés aux élèves. Les compétences ont aussi leur rôle dans l'explication de ce constat: si l'usage de l'ordinateur pour créer des documents courts à l'aide du traitement de texte est assez répandu, les compétences limitées dont les stagiaires font preuve concernant l'édition de documents longs, le traitement des images numériques, et plus généralement la production de documents multimédias peuvent sans doute limiter leurs capacités à produire des documents pédagogiques.

Bien que l'accès aux ordinateurs dans les établissements scolaires semble généralement possible, en particulier en lycée et en collège, nous notons finalement la faible utilisation des logiciels en classe qui représente donc le cadre d'usage le plus contraint. L'utilisation de logiciels disciplinaires se développe, mais l'usage $\mathrm{du}$ tableur reste confidentiel, ce qui est particulièrement préoccupant pour les scientifiques puisque l'usage de ce logiciel est préconisé par les programmes du second degré.

L'usage des technologies chez les stagiaires PLC semble fortement lié au lieu de stage et plus particulièrement à la personne ressource lorsqu'elle existe. Ce rôle de personne ressource semble moins important chez les stagiaires PE, qui s'adressent plus volontiers à leurs pairs. Les données recueillies semblent indiquer que l'IUFM joue finalement très partiellement le rôle d'initiateur auquel l'institution est en droit de s'attendre. Lorsque la formation est directement en relation avec les usages des stagiaires, son réinvestissement est assuré quel qu'en soit le cadre. Comme nous l'avons mentionné (Reims), la modification d'un dispositif de formation excluant une formation multimédia se traduit par une diminution des compétences. A contrario, une formation en TIC non reliée à un usage sera peu réinvestie, voire totalement oubliée par les stagiaires, ce qui s'accompagne d'un fort sentiment d'autodidaxie. Ceci se vérifie à Dijon où ce sentiment d'autodidaxie prédomine alors que la formation aux technologies est intégrée dans les modules obligatoires proposés en licence par l'université et dans la formation de première année à l'IUFM (PE).

En conclusion, cette étude nous fait apparaitre une population utilisant les technologies de façon assez basique, qui ne se montre pas opposée à l'introduction des TICE dans leur enseignement. Cependant, il semble que le réinvestissement des connaissances personnelles en classe est limité, marquant les contraintes normatives comme le respect des programmes de la discipline enseignée et une formation certainement insuffisante. Il existerait bien, comme nous l'avions proposé, des écarts qualitatifs entre les usages d'un même outil dans les différents cadres. Pour dépasser ces difficultés, il nous semble important de conduire des actions de formation fortement reliées aux usages en classe.

\section{Références}

Abboud-Blanchard, M., Lagrange, J.-B., Le Borgne, P. et Parzysz, B. (2005). Appropriation des outils TIC par les stagiaires IUFM et effets sur les pratiques professionnelles. Dans les Actes du colloque InterIUFM. Former des enseignants professionnels, savoirs et compétences. Nantes: Institut Universitaire de Formation des Maîtres des Pays de la Loire.

Baillat, G. et Vincent, J. (2003). Les TICE et les jeunes enseignants: les représentations en formation initiale. Dans J.-B. Lagrange, M. Artigue, D. Guin, C. Laborde, D. Lenne et L. Trouche (dir.), Actes du colloque européen ITEM: intégration des technologies dans l'enseignement des mathématiques. Institut Universitaire de Formation des Maîtres de Reims, France. Récupéré le 7 septembre 2006 de l'archive EduTice, http://archivetematice.ccsd.cnrs.fr.

Baron, G. L., Bruillard, E. et Levy, J.-F. (2000). Les technologies dans la classe, de l'innovation à l'intégration. Paris: Institut National de Recherche Pédagogique [INRP] et Association Enseignement Public et Informatique [EPI].

Baron, G. L., Caron, C. et Harrari, M. (2005). Le multimédia dans la classe à l'école primaire. Lyon: Institut National de Recherche Pédagogique.

Bellemain, F. et Capponi, B. (1992). Spécificité de l'organisation d'une séquence d'enseignement lors de l'utilisation de l'ordinateur. Educational Studies in Mathematics, 23(1), 59-97.

C2i niveau 2 - Enseignant. (n.d.). Récupéré le 28 juin 2006 du site du 
Ministère de l'éducation nationale, de l'enseignement supérieur et de la recherche, http://www2.educnet.education.fr/sections/formation/ certification/c2i-ens.

Chevallard, Y. (1997). Familière et problématique, la figure du professeur. Recherche en didactique des mathématiques, 19(2), 221-266.

Laborde, C. et Capponi, B. (1994). Cabri-géomètre constituant un milieu pour l'apprentissage de la notion de figure géométrique. Recherche en didactique des mathématiques, 14(1), 165-209.

Lagrange, J.-B., Lecas, J.-F. et Parzysz, B. (sous presse). Les professeurs stagiaires d'IUFM et les technologies. Quelle instrumentation? Recherche et Formation.

Lenfant, A. (2003). De la position d'étudiant à la position d'enseignant: l'évolution du rapport à l'algèbre de professeurs stagiaires. Thèse de doctorat, Institut de Recherche sur l'Enseignement des Mathématiques de l'Université Paris 7 - Denis- Diderot.

Linard, M. (1996). Des machines et des hommes: apprendre avec les nouvelles technologies. Paris : L'Harmattan.

Ministère de l'éducation nationale, de l'enseignement supérieur et de la recherche. Textes de référence des B2i niveaux 1,2 et 3. (n.d.). Récupéré le 28 juin 2006 du site du ministère http://www2.educnet.education. fr/sections/formation/.

Monaghan, J. (2004). Teachers' activities in technology-based mathematics lessons. International Journal of Computers for Mathematical Learning, 9(3), 327-357.

Poncet, P. et Régnier, C. (2003). Les TIC:éléments sur leurs usages et effets (Note évaluation 03.01). Vanves, France: Direction de l'évaluation et de la prospective du ministère de l'Éducation nationale. Récupéré le 7 septembre 2006 du site du ministère de l'Éducation nationale de la France, ftp://trf.education.gouv.fr/pub/edutel/dpd/noteeval/ ne0301.pdf.

Programmes officiels de l'école primaire, du collège et des lycées. (n.d.). Récupéré le 28 juin 2006 du site du Ministère de l'éducation nationale, de l'enseignement supérieur et de la recherche, http://eduscol. education.fr/D0048/.

Rinaudo, J.-L. (2001). Le rapport à l'informatique des enseignants de l'école primaire. Thèse de doctorat non publiée, Université Paris X Nanterre.

\section{Notes}

1 Les lecteurs pourront se référer aux programmes officiels de l'école primaire, du collège et des lycées du Ministère de l'éducation nationale, de l'enseignement supérieur et de la recherche (Programmes officiels de l'école primaire, du collège et des lycées, n.d.) ou aux textes de référence des B2i niveaux 1, 2 et 3 (Textes de référence des B2i, n.d.).

2 Cette terminologie officielle regroupe les technologies permises par l'ordinateur et les réseaux.

3 Un rapport (Poncet et Régnier, 2003) de la direction de l'évaluation et de la prospective (DEP) du ministère de l'Éducation nationale montre les réticences de nombreux enseignants, au-delà d'une frange déjà " engagée ".

${ }_{4}$ Technologies de l'information et de la communication pour l'enseignement.

5 Le Certificat Informatique et Internet niveau 1 (C2i n1, http://c2i.education.fr/) atteste la maîtrise d'un ensemble de compétences nécessaires à l'étudiant pour mener les activités qu'exige aujourd'hui un cursus d'enseignement supérieur.

${ }^{6} 42 \%$ des Français sont équipés en février 2003 (enquête CSA menée pour le ministre délégué à l'industrie).

${ }^{7}$ Logo délivré par le ministère de l'Éducation nationale. Il permet d'identifier les créations multimédias qui, après expertise par des enseignants et des spécialistes du domaine, répondent aux besoins et aux attentes du système éducatif.

${ }^{8}$ En France, le cursus à l'école primaire est organisé en trois cycles (cycle 1 ou cycle des apprentissages premiers, cycle 2 ou cycle des apprentissages fondamentaux et cycle 3 ou cycle des approfondissements) pour permettre à chaque enfant de parcourir sa scolarité de manière continue sans redoublement (décret du 14 mars 1995). 